\title{
Research of method for detection of rail fastener defects based on machine vision
}

\author{
Zhenzhen WANG $^{1, b}$, siming WANG ${ }^{1, a}$ \\ ${ }^{1}$ School of Automation \& Electrical Engineering, Lanzhou Jiaotong University, Lanzhou, China \\ 730070. \\ a15769349708@163.com, b3343750036@qq.com
}

Keywords: railway fasteners, Canny operator, Hough transform, fusion of LBP and HOG, SVM.

\begin{abstract}
The detection of rail fastener defects is the key to ensure the running safety of high-speed trains. Traditional method is usually be detected rely on train workers who walk along railway lines to find out the potential risks. The method by artificial maintenance is slowly, costly, and dangerous. As to solve the problem, an automatic detect method based on machine vision is proposed for all kinds of rail fastener defects. background subtraction algorithm is used to achieve the accurate positioning of railway fasteners in this paper. The method is based on video sequence for processing rail fastener image. First of all, realized rail fastener accurate positioning based on the improved Canny operator and the Hough transform to extract linear; Then, extracted the characteristics of fastener defect feature by fusion LBP (Local Binary Pattern, Local Binary Pattern) and HOG (Histogram of Oriented Gradient direction Gradient Histogram); Finally, the characteristics of Histogram as the SVM (Support Vector Machine SVM) input values is used to buckle defect classification. The experimental results show that the method compared with the traditional single classification method, has higher real-time and accuracy, and can meet the requirement of rail fastener defect detection.
\end{abstract}

\section{Introduction}

All manuscripts must be in English, also the table and figure texts, otherwise we cannot publish your paper. Please keep a second copy of your manuscript in your office. When receiving the paper, we assume that the corresponding authors grant us the copyright to use the paper for the book or journal in question. Should authors use tables or figures from other Publications, they must ask the corresponding publishers to grant them the right to publish this material in their paper. Use italic for emphasizing a word or phrase. Do not use boldface typing or capital letters except for section headings (cf. remarks on section headings, below).

Do not number your paper: All manuscripts must be in English, also the table and figure texts, otherwise we cannot publish your paper. Please keep a second copy of your manuscript in your office. When receiving the paper, we assume that the corresponding authors grant us the copyright to use the paper for the book or journal in question.

Rail fastener defect detection is the key to ensure the high speed railway train transportation safety. With the development of high-speed and heavy-duty train, traditional method by manual detection can not meet the needs of the safety for train running. Based on the development of the image and pattern recognition technology, automatic detection of railway fasteners defects has become possible. In 2009, our country launched the " 836 " project plan focus on research and development of ultra high speed railway train. Automatic detection of rail fastener defects is the important part in this plan [1]. Nowadays, foreign technology about automatic detection of rail fastener defects is relatively mature. But the type is different with rail fasteners in our country, the method is not applicable to our railway system. In our country about the study is still a preliminary research. Hao Feng et al.[2] put forward to determine the location to the rail surface position and orientation to the sleeper position based on fasteners' mutual information. Then, they calculated the defective fasteners by using template matching method based on gray level and gradient distribution characteristics of rail surface area. Haibo Zhang et al. [3, 4] realize the state of the fastener pattern classification by using a structured light on fasteners. The process includs the line search method, etc. . Yang Jinfeng et al. [5], first, 
presented a new algorithm about detecting parallel lines. It is used to detect fasteners positioning. Then, they uses the oriental field algorithm to identifying the target fastener state based on statistical information. The algorithm reduced the dependence on image quality, but it had the higher requirements to the fastener template. Although, there are some studies about this, it still can not meet the requirement of detecting about rail fasteners defects.

Aimed to solve the question, a new automatic method was presented base on machine vision. The new method has the advantage of non-contact, high speed, high accuracy, high intelligence, high adaptability, etc. Based on the existing research results about fastener detection, the system need to solve the overall design of the detection system, and the algorithm for detecting, recognizing, classifying fasteners. The overall design of the detection system flow chart is given as Fig.1.

$\begin{gathered}\text { Captured } \\ \text { video }\end{gathered} \rightarrow \begin{gathered}\text { Fastener } \\ \text { positioning }\end{gathered}$
posions $\rightarrow \begin{gathered}\text { Image } \\ \text { processing }\end{gathered} \rightarrow \begin{gathered}\text { Extracting } \\ \text { characteristic }\end{gathered} \rightarrow \rightarrow \begin{gathered}\text { Fastener } \\ \text { classification }\end{gathered} \rightarrow \begin{gathered}\text { Save \& } \\ \text { alert }\end{gathered}$

Fig.1 Flow chart of overall design for the detection system

The new method realize the precise localization of fasteners based on the improved Canny algorithm combined with extracting straight line rely on Hough transform. All various kinds of images under different working conditions were gotten by the camera under the train. Fusion LEP and HOG features were extracted as the input values of SVM. The SVM classifier is trained by offline. Then, we can get the accurate classification of the positive fasteners and the negative fasteners. The experiment shows that an average of $98.9 \%$ precision has been achieved for detecting and classifying the detective rail fasteners. In addition, the proposed algorithm is more robust than several mainstream methods and can meet the need of automatic inspection of fastener defects.

The video collecting and processing system is as Fig.2.

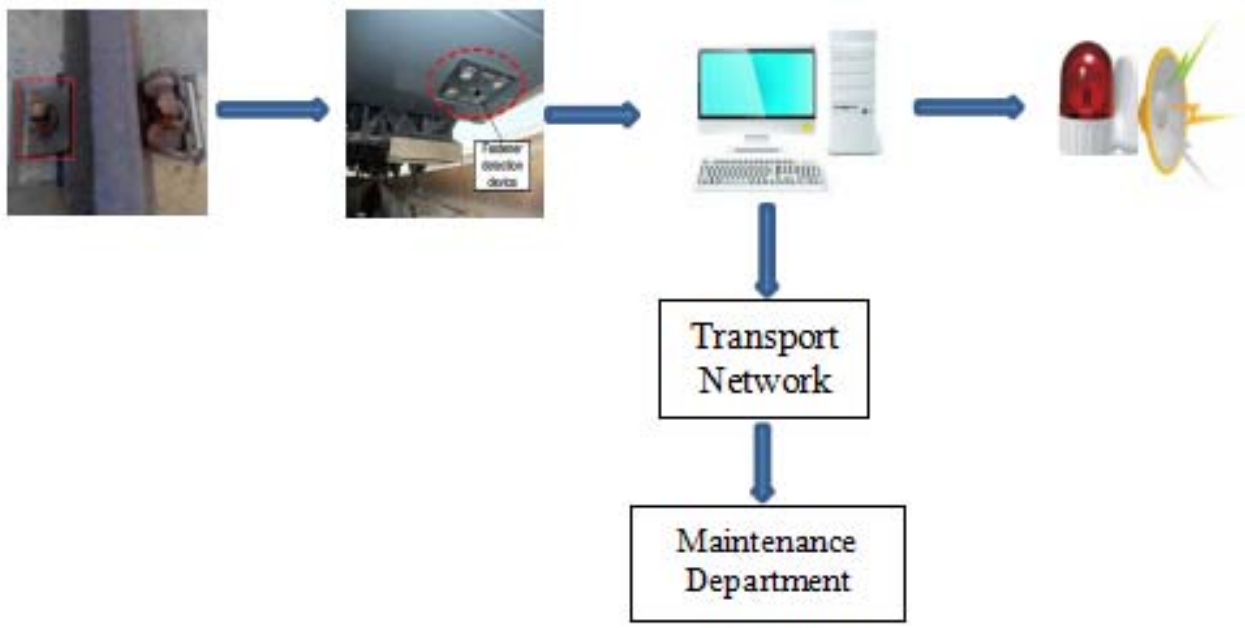

Fig.2 Flow chart of fastener detection system

\section{Fasteners positioning}

Accurate positioning of fasteners is the key to detect the real edge information of rail and fasteners. The common edge detection algorithms are Sobel, Laplace, Canny. Canny operator compared with other operators. It can obtained the complete edge information and get better balance between noise suppression and edge information. However, hysteresis threshold is the disadvantage of Canny operator. So improved Canny edge detection operator was adopted in this paper.

The theory of improved Canny edge detection operator.

Now various kinds of high-speed track inspection cars have been developed. The video image was captured by onboard camera. The key of this paper is to improve the image processing algorithms based on existing algorithms. Because fasteners' edge characteristic is obvious and main characteristics. We need to acquire the edge characteristics. Edge detection is the foundation of other processes. Canny operator for edge detection is the best method to obtain the complete information. Dynamic threshold of Canny was selected to make sure the adjustable and accurately edge. Then we can extract straight-line based on improved Hough transform to realize the accurate fastener 
positioning. The basic principle of Canny operator [6] used gaussian filter to smooth images by the convolution operation, and look for local maximum values of the image gradient to determine the edge. In general, algorithm can realize edge detection with double threshold detection and edge connection. However, the existence of false edge detection effect and noise double threshold setting can not effectively remove the false edge.To take into account the image threshold transform, this paper the accurate edge information by using edge subtraction based on Canny double threshold algorithm. Its principle is used two kinds of threshold to detect the edge information. First, we can set the high and low threshold to get the complete information of fasteners and steel rail based on the gray-level histogram. The image was labeled Cannyl. Then, the noise information of images were gotten by setting the high and low threshold based on the gray-level histogram. The image was labeled Canny2. Finally, we can get the accurate edge information by subtraction operator between Cannyland Canny2. The key of operator is to accurately calculate gray-level histogram.

\section{Extracting straight line based on Hough transform.}

To extract straight line of the edge image based on Hough transform. The basic principle of Hough transform [7] is point-line duality. Considering the point-line duality rather wasteful device, it was replaced the slope-intercept formulation with the so-called "normal" $(\rho, \theta)$ form for the straight line:

$$
\rho=x \cos \theta+y \sin \theta
$$

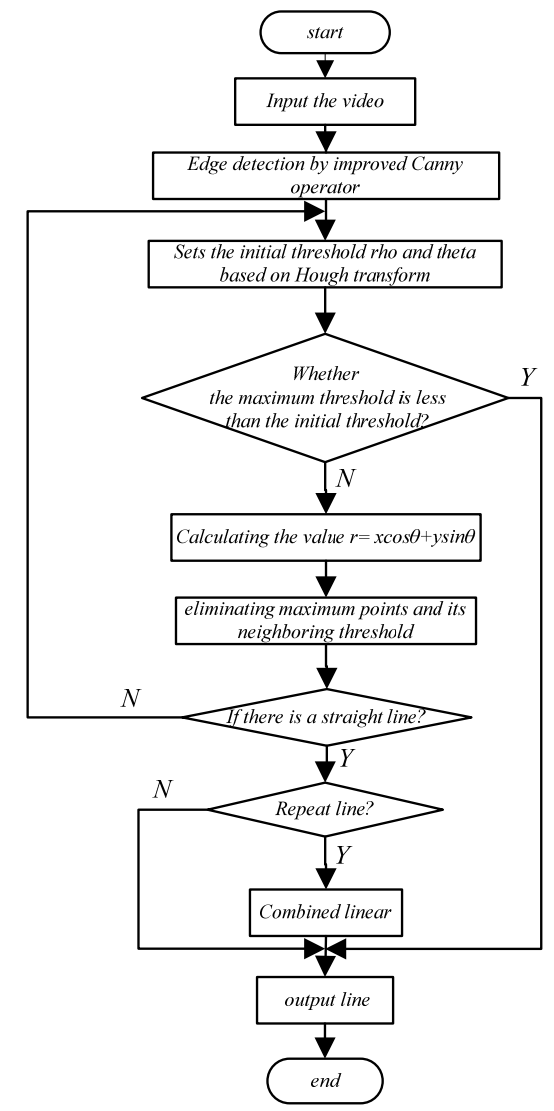

Fig.3 Flow chart of rail fasteners positioning

First, we should initialize the transform threshold $\rho$ and $\theta$. Then, searching biggest image threshold compared with the initial threshold. When the biggest image threshold is less than the initial threshold. The process goes to the end; If the biggest image threshold is bigger than the initial threshold. We can calculate $\gamma$ value by the formula : $\gamma=x \cos \theta+y \sin \theta$ and remove the maximum of pixels and its neighborhood. Finally, by judging whether there is a linear and whether the linear is repeated. If the linear is not existent, the program will start a new cycle to search biggest image threshold. If the linear is existent and is not repeated, the program will directly output the linear. At this time, rail fastener area can be gotten. The flow chart of rail fasteners positioning is shown as Fig.3. The result of positioning is given as Fig.4. 

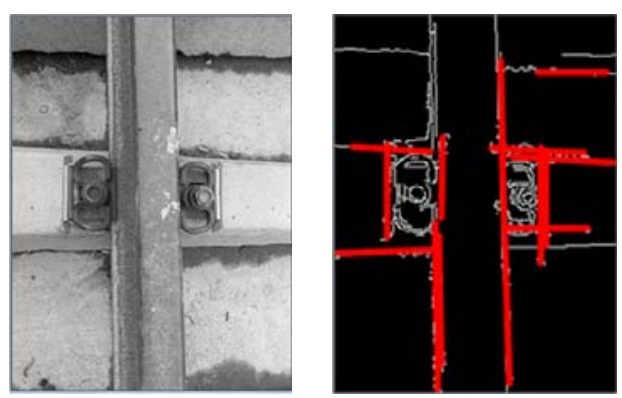

Fig.4 Original image and the positioning result

\section{Extracting Feature of rail fasteners}

Extracting Fasteners feature [8-12] is the foundation of recognizing and classifying fastener defects. In this paper, considering the characteristics of the fasteners, the advantages and disadvantages of HOG feature and LBP feature were analyzed. Finally, fusion of LBP and HOG feature was adopted to recognize and classify fastener defects.

\section{Extracting LBP Feature.}

LBP $[13,14]$ (Local Binary Pattern, Local Binary Pattern) is a kind of operator which used to describe the Local texture feature of the image.

It initially defined within the window of $3 * 3$. The center pixel was used as the basal threshold. Compared the adjacent eight pixel gray value with the basal threshold value. When the adjacent eight pixel gray value is greater than the center pixel value, it will be marked as 1 . Otherwise, it will be marked as 2 .

The simple computing method and the good detection effect are the advantage of this operator. Besides, the operator is not sensitive to light. But it is more sensitive to noise. The schematic diagram is shown as Fig.5. The original image and the results are shown as Fig.6 and Fig.7.

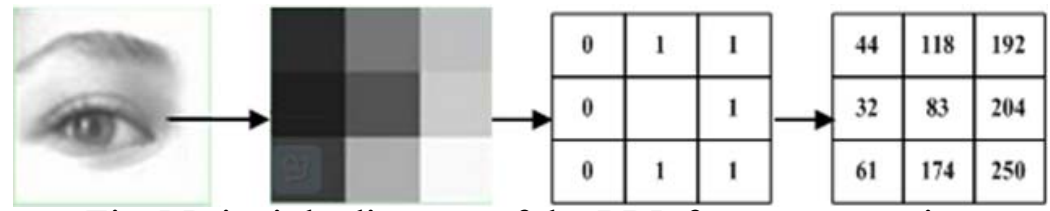

Fig.5 Principle diagram of the LBP feature extraction

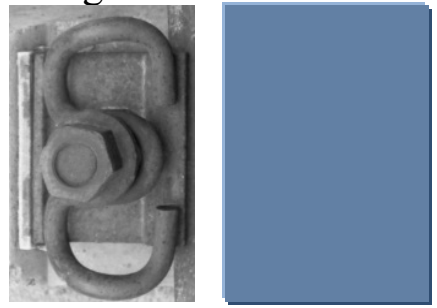

Fig.6 Original image and the result image of intact fasteners

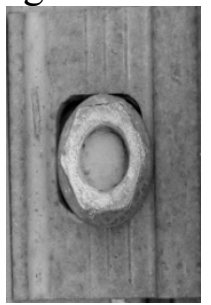

Fig.7 Original image and the result image of defective fasteners

\section{Extracting HOG Feature.}

HOG [15] (Histogram of Oriented Gradient) detects the gradient direction histogram feature by calculating and statistical image gradient direction histogram to form characteristics of local area.The operator can be partially offset the effects of the change of illumination. But its generation process is long. So it leads to a poor real-time performance.For the real time 
requirement of fasteners defect detection, the operator is not considered as a classification characteristic value alone. In this paper, the result of extracting HOG feature under various working conditions were shown as Fig.8.

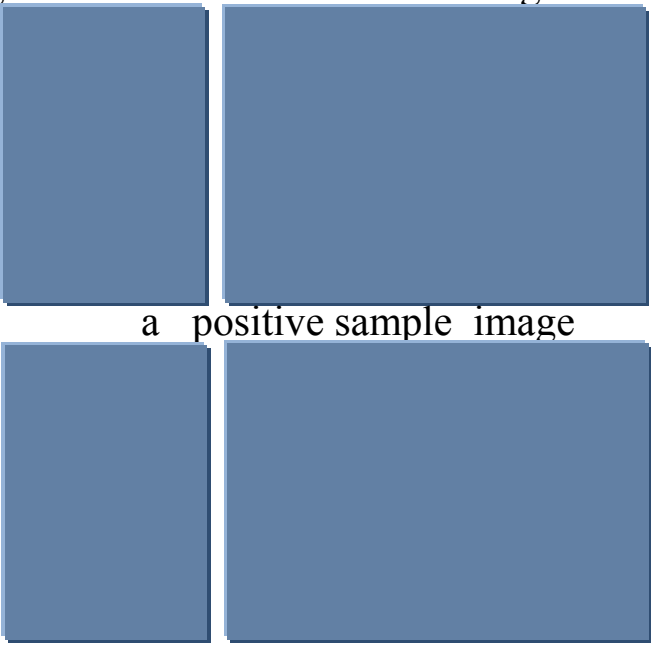

c illumination sample image

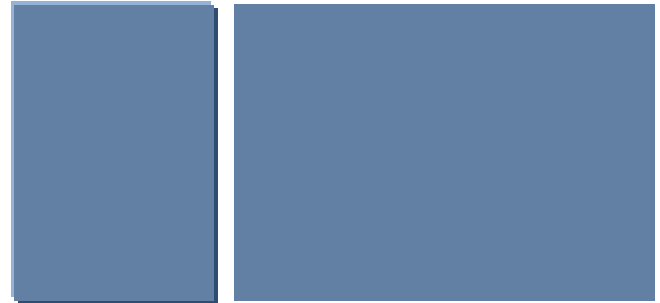

b negtive sample image

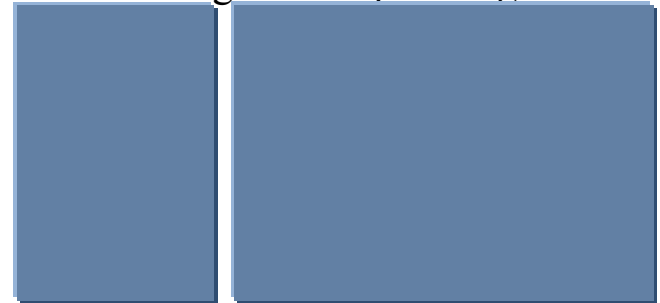

d distractors sample image

Fig. 8 Fastener original image and the result image under various working conditions

\section{Extracting fusion of LBP and HOG features.}

In view of the advantages and disadvantages of LBP and HOG features and fasteners special edge character, the fusion of LBP and HOG feature[16] was used to extract different types of fasteners characteristic value. The characteristic value will be as the input of the SVM to realize the classification of fasteners defects. Fastener original image and the inspection result images by the fusion of LBP and HOG feature under various working conditions were shown as Fig. 9 and Fig. 10.

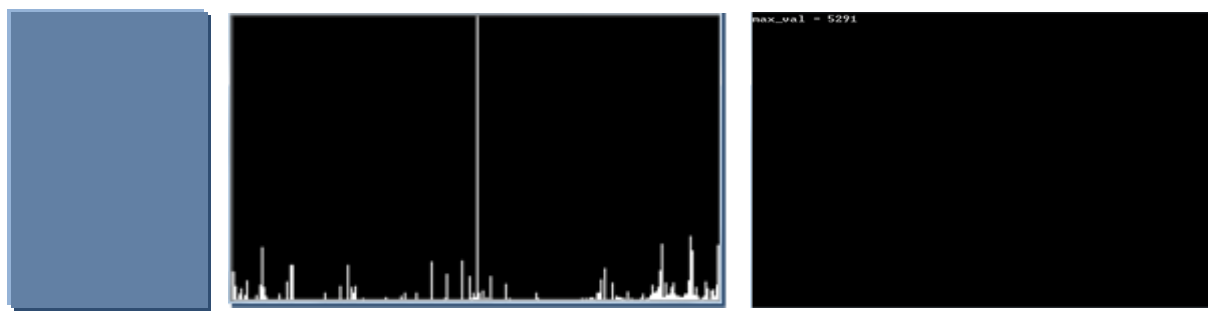

Fig.9 Fasteners LBP features image and the result by fusion of LBP and HOG feature of intact fasteners
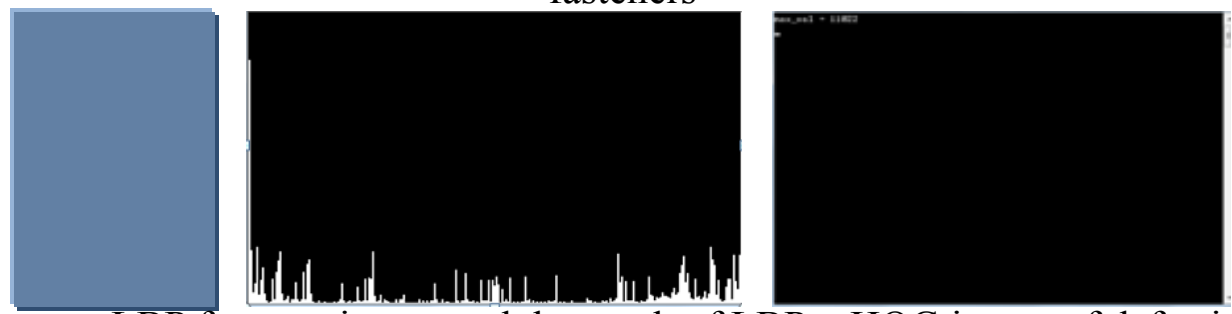

Fig.10 Fasteners LBP features image and the result of LBP + HOG image of defective fasteners

\section{Analysis of experimental results}

Support Vector Machines ( SVM) [17]theory is proposed for binary classification.The classifier of fusion of many features can be founded by combining multiple binary classification on the basis of the Support Vector Machine (SVM).Considering the fasteners defect characteristics, this paper adopts the classifier of fusion of many features algorithm to classify fasteners defects. First, the main classifier were trained by 5000 positive samples pictures and 1000 negative samples pictures collected to establish the main training images. Then, randomly selected 2000 pictures was used as the input of the SVM to classify the fasteners defects. The detailed procedure was shown as follows: 
1) calculating the LBP features of images.

2) to extract HOG feature based on the LBP images.

3) training the SVM for getting the initial classifier.

4) using the trained classifier for realizing the images classification.

In this part, parts of the positive sample images and the negative sample images under different weather and environment were given as Fig.11.
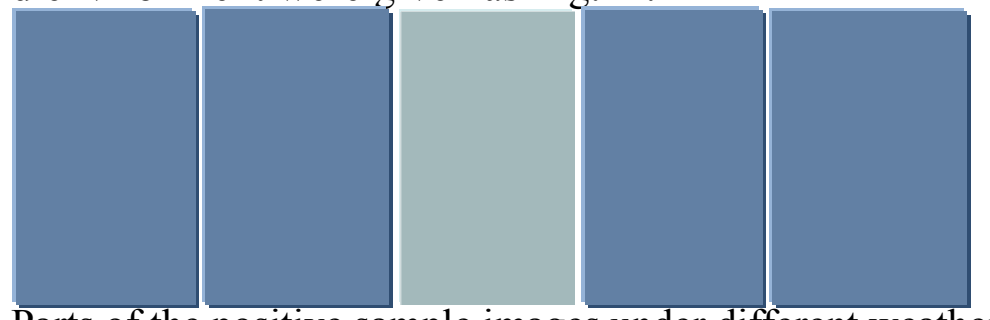

a Parts of the positive sample images under different weather

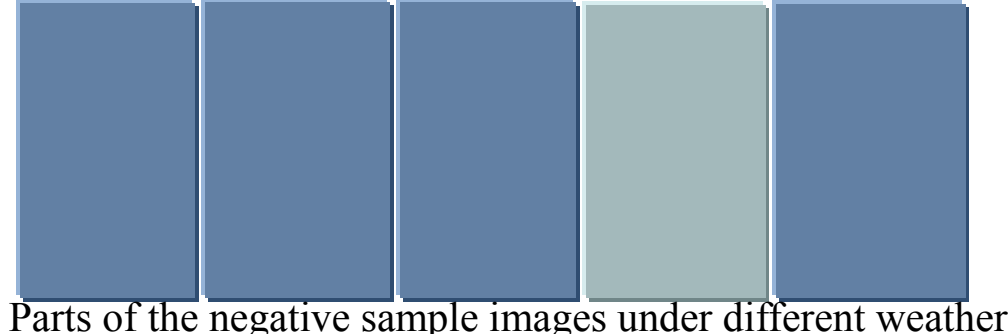

b Parts of the negative sample images under different weather

Fig. 11 Part of the sample picture under various working conditions

We compared the different characteristics of the classifiers algorithm in the different computer. The results are shown in Table 1.

The experimental results revealed that: the accuracy and real-time performance of our method than the other two methods has been effectively improved. Different computer configuration affect the real-time detection. Because of the limitation of laboratory, the speed of inspection is to be solved for future study on this problem. But I hope it can have a certain reference value for future scholars to research.

Table 1 Performance comparison of the different characteristics of classification algorithm

\begin{tabular}{cccc}
\hline Type & LBP+SVM & HOG+SVM & LBP+HOG+SVM \\
\hline CPU,4G RAM Testing time(s) & 0.0412 & 0.0392 & 0.0376 \\
CPU,2G RAM Testing time(s) & 0.0541 & 0.0452 & 0.0416 \\
Accuracy & $97.2 \%$ & $96.7 \%$ & $98.9 \%$ \\
\hline
\end{tabular}

\section{Conclusion}

Rail fastener defect detection method based on machine vision research.First of all, set up a kind of based on the improved Canny edge detection and Hough transform line extraction method, realize accurate positioning of fasteners. This method is affected by the weather and other small.Then using the fusion of LBP and HOG features as the input of the SVM to realize accurate fastener defects recognition and classification, improved the buckle defect recognition rate.Through the above theoretical and experimental results it can be seen that the method has achieved good results in this paper.In the follow-up research work, in order to achieve rapid detection and high accuracy, first of all, to further improve the existing algorithms, improve the efficiency of the algorithm.Able to draw lessons from other university research results inspected platform is the key point of next work.

\section{References}

[1]. Xu Guiyang, Shi Tianyun, Ren Shengwei, et al. Development of the On-board Track Inspection System Based on Computer Vision. China Railway Science.Vol.4(2013) No.1, p. 139-144.

[2]. Hao Feng, Zhiguo Jiang, Fengying Xie, et al. Automatic Fastener Classification and Defect Detection in Vision-Based Railway Inspection Systems. IEEE transactions on instrumentation and 
measurement. Vol.63(2014) No.4, p. 877-888.

[3]. Rong Zou, Zhenying Xu, Jinyang Li,et al. Real-time monitoring of brake shoe keys in freight cars. Proceedings of the Institution of Mechanical Engineers Part F Journal of Rail and Rapid Transit. Vol.16(2015) No.3, p.191-204.

[4]. Zhang Haibo, Yang Jinfeng,Tao Wei, et al. Vision method of inspecting missing fastening Components in high-speed railway. Applied Optics. Vol.50(2011) No.20, p.3658-3665.

[5]. Jinfeng Yang, Wei Tao, Manhua Liu, et al. An efficient direction field-based method for the detection of fasteners on high-speed railways. Sensors. Vol.11(2011)No.8, p.7364-7381.

[6]. J. Canny. A computational approach to edge detection. IEEE Transactions on Pattern Analysis and Machine Intelligence. Vol.8(1986)No.6, p.679-698.

[7]. Zhang Jiangxin, Shen Xiaolan, Wang Hui, et al. Fast multiline detection algorithm using randomized Hough transform . Journal of Zhejiang University of Technology, Vol.41(2013)No.3, pp. 344-350.

[8]. Vila, M., Bardera, A., Xu, Q., et al. Tsallis entropy-based information measures for shot boundary detection and key frame selection. Signal Image Video Process. Vol.7(2013) No.3, p. 507-520.

[9]. Yu Jing, Qin Zengchang, Wan Tao, et al. Feature integration analysis of bag-of-features model for image retrieval. Neurocomputering. Vol.120(2013) No.23, p. 355-364.

[10]. P. Ghamisi and J. A. Benediktsson. Feature selection based on hybridization of genetic algorithm and particle swarm optimization. IEEE Geosci. Remote Sens. Lett.. Vol.12(2015)No.2, p. 309-313.

[11]. P. Ghamisi, J. A. Benediktsson, G. Cavallaro,et al. Automatic framework for spectral-spatial classification based on supervised feature extraction and morphological attribute profiles. IEEE J. Sel. Topics Appl. Earth Observ. Remote Sens.. Vol.7(2014) No.6, p. 2147-2160.

[12]. P. Ghamisi, J. A. Benediktsson, J. R. Sveinsson. Automatic spectral-spatial classification framework based on attribute profiles and supervised feature extraction. IEEE Trans. Geosci. Remote Sens.. Vol.52No.9(2014), p.5771-5782.

[13]. Suruliandi A, Meena K, Reena Rose R. Local binary pattern and its derivatives for face recognition. iet Computer Vision, Vol.6(2012)No.5, p.480-488.

[14]. Gao Tao, He Mingyi, Dai Yuchao, et al. Face recognition using multi-level histogram sequence local binary pattern. Journal of Image and Graphics. Vol.14(2009)No.2, p.202-207.

[15]. Chen Rui, Peng Qiming. Pedestrian detection based on HOG of stable area. Journal of Computer-Aided Design \& Computer Graphics.Vol.24(2012) No.3, p.372-377.

[16]. Xiang Zheng, Tan Henhliang, Ma Zhengming. Performance comparison of improve HOG, Gabor and LBP. Journal of Computer-Aided Design \&Compute Graphics. Vol. 24(2012) No.6, p.788-792.

[17]. LIU Jiajia, LI Bailin, LUO Jianqiao, et al. Railway Fastener Detection Algorithm Integrating PHOG and MSLBP Features. Jounal of southwest jiaotong university. Vol.50(2015)No.2, p.256-263. 